\title{
Retention of Stroke Education Provided during Hospitalization: Does Provision of Required Education Increase Stroke Knowledge?
}

\author{
Brenda Johnson $^{a} \quad$ Diane Handler $^{b}$ Victor Urrutia $^{a} \quad$ Anne W. Alexandrov ${ }^{c, d}$ \\ aDepartment of Neurology, College of Medicine, John Hopkins Medical Center, Baltimore, \\ MD, USA; bStroke Team, Unity Point Health - St. Luke's Hospital, Cedar Rapids, IA, USA; \\ 'Department of Neurology and Mobile Stroke Unit, College of Medicine, College of Nursing,

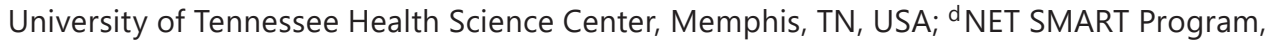 \\ Health Outcomes Institute, Fountain Hills, AZ, USA
}

\section{Keywords}

Stroke education $\cdot$ Acute stroke $\cdot$ Learning retention $\cdot$ Pilot studies

\begin{abstract}
Background: Provision of stroke education to patients is a Centers for Medicare/Medicaid (CMS) requirement. However, little is known about retention of the educational content. Methods: Two pilot studies were conducted: Pilot A delivered CMS-required stroke education during hospitalization in a standardized manner and tested knowledge retention in patients returning to the Stroke Clinic for 1-month follow-up; Pilot B randomized patients to either a control group with standardized education or a test-enhanced learning group (target), with measurement of stroke knowledge retention at hospital discharge. Results: A total of 198 patients/caregivers participated in Pilot $A$, with only $25 \%$ scoring $100 \%$ correct on required stroke education items. The question most commonly answered incorrectly ( $n=117 ; 59 \%)$ was "personal risk factors for stroke," and 74 (37\%) could not correctly identify stroke signs and symptoms. Pilot B found that significantly more target group patients could identify their personal stroke risk factors ( $100 \mathrm{vs.} 67 \% ; p=0.04$ ) and the purpose of their secondary prevention medications ( 87 vs. $40 \% ; p=0.02$ ) compared to controls. Discussion: While stroke education is required during hospitalization, its ability to produce retention may be poor. We propose study of test-enhanced learning methods through the Targeted Education in Stroke Trial (TEST) to examine the effect of novel teaching methods on patient/caregiver knowledge retention.




\section{Introduction}

An acute stroke event produces profound physiological, psychological, and socioeconomic challenges for both patients and their family members [1-8]. The need to learn new information about what stroke is, the causative pathogenesis and additional personal risk factors for stroke, signs and symptoms of stroke, and medications and other treatments to manage and prevent future events constitute significant learning needs that nurses and other providers are required to respond to before hospital discharge $[9,10]$. With a US average hospital length of stay of approximately 5 days [11], the responsibility to increase patients' and family members' knowledge of stroke is daunting.

Numerous studies have shown that adults learn best when there is a perceived need and an opportunity to process, apply, question, and reeducate when an evaluation of learning determines need [12-18]. However, hospital environments rarely provide optimal conditions that facilitate learning, and when combined with a new stroke event, the likelihood of learning is further challenged by anxiety and fear [19-22]. Furthermore, patients are often poor candidates for education retention due to disabilities produced by the stroke event, and while families may be better positioned to retain educational information, the sudden changes produced by stroke in their affected family member often alter both attentiveness and recall of information [19, 22].

Despite environmental and situational limitations affecting learning, provision of stroke education for patients and family members is a Stroke Center requirement $[9,10]$. However, little is known about patients' and family members' retention of required stroke educational elements following hospital discharge when this information is of most importance. Therefore, we undertook a two-phase pilot study to determine retention of stroke education content at 4 weeks after hospital discharge (Pilot A), and to examine the feasibility and signal of efficacy for use of test-enhanced learning methods (Pilot B) in an acute stroke population.

\section{Methods}

Institutional Review Board approval was obtained for the conduct of an observational study of stroke knowledge retention at a community hospital in the Midwest USA, and the conduct of a randomized experimental pilot of test-enhanced learning at an inner-city mid-Atlantic academic center. Four written test questions were developed and edited to a seventh-grade learning level (Table 1), with each question addressing Centers for Medicare and Medicaid (CMS)-required educational elements [10]: recognition of stroke signs and symptoms, knowledge of personal stroke risk factors, 911 utilization, and understanding prescribed medications to prevent stroke. An additional test item dealing with understanding the "type of stroke" the patient suffered was added to the Pilot B study. In both pilot studies, test items dealing with stroke risk factors and prescribed medications were developed so that they could capture content personalized to each subject enrolled in the study.

Standardized stroke education focused on the CMS-required items was provided at both sites according to routine Stroke Team protocols using methods approved by stroke center certifying bodies on recent certification reviews. Verbal information exchange was supplemented by additional materials to reinforce learning, including stroke educational brochures and other personalized handouts, along with time for questions and answers for all stroke service patients at both study sites. Subjects in Pilot B also received education on the "type of stroke" they had suffered, along with all other CMS-required educational elements. Patients and primary caregivers were encouraged at discharge to bring their written materials home as additional resources to support learning. Both pilot studies recruited a convenience sample of adult English-speaking, literate patients and/or primary caregivers.

Pilot A Methods

Pilot A was a prospective observational study that aimed to understand stroke knowledge retention at 30 days \pm 14 days from the time of receipt of CMS-required hospital education. Patients and primary caregivers were recruited for the study when they returned to the Stroke Clinic for a postdischarge 1-month 
Table 1. Written test questions used in Pilots A and B

Question $1^{\mathrm{a}} \quad$ The type of stroke I had was:

A. Ischemic stroke (clogged artery in the brain)

B. Brain hemorrhage (burst artery in the brain)

Question 2 Circle the 6 stroke signs and symptoms listed below:

A. Sudden weakness of the face, arm and/or leg

B. Sudden onset of chest pain

C. Sudden vision loss in one or both eyes

D. Sudden change in sensation in face, arm and/or leg

E. Sudden onset of fever

F. Sudden onset of breathing difficulty/shortness of breath

G. Sudden onset of dizziness

H. Sudden onset of nausea and vomiting

I. Sudden onset of worst headache of your life

J. Sudden difficulty findings the correct words to say and/or slurred speech

Question 3 If you or someone else has stroke symptoms, what is the best first response? (circle only one)

A. Lie down and wait to see if symptoms go away

B. Call the doctor's office for advice

C. Contact family or friends to help

D. Drive yourself or the patient to the hospital

E. Call an ambulance (911)

Question $4 \quad$ My stroke risk factors include... (circle all that apply)

A. High blood pressure

B. Diabetes

C. Smoking

D. High cholesterol

E. My previous stroke

F. Atrial fibrillation

G. My previous heart attack

H. Heart failure

I. Abnormal hole in my heart

J. Blood that clots too much

K. Clogged neck artery

L. Abnormal arteries in the brain that easily bleed

M. Overweight by $\geq 20 \mathrm{lb}$

N. Physical inactivity

O. Excessive alcohol use

P. Illegal drug use

Q. Family members with stroke

Question 5 The medication that I take to prevent a future stroke includes which of the following? (circle all that apply)

A. Medication to keep blood from clotting

B. Medication to control blood pressure

C. Medication to lower cholesterol

D. Medication to control blood sugar

E. Medication to help me quit smoking

\footnotetext{
${ }^{\mathrm{a}}$ Question 1 was only used in Pilot B.
} 
Table 2. Pilot A stroke knowledge test results

\begin{tabular}{lll}
\hline $\begin{array}{l}\text { Test item } \\
\text { No. }\end{array}$ & Knowledge tested by test item & $\begin{array}{l}\text { Subjects scoring "item correct," } \\
n(\%)\end{array}$ \\
\hline 1 & Emergent response to stroke & $178 / 198(90)$ \\
2 & Recognizing signs and symptoms of stroke & $125 / 198(63)$ \\
3 & Personal risk factors for stroke & $81 / 198(41)$ \\
4 & Medications to prevent a future stroke & $147 / 198(74)$ \\
\hline
\end{tabular}

follow-up appointment, and those consenting to participate were then given a written test consisting of the 4 test questions (Table 1) to determine retention of stroke education presented during hospitalization. Because patients with their primary caregivers were the target of the Stroke Team's hospital education protocol, those patients that returned with their primary caregiver were allowed to complete the test together if the family member attested to receiving education during hospitalization. Tests were graded in person with consenting subjects, and items that were incorrect triggered delivery of additional discussion and one-on-one education with the Stroke Team's advanced practice nurse.

Study demographic data were limited to age, sex, and causative pathogenesis of stroke to limit confidentiality and privacy risks in the sample. Data were entered and analyzed in SPSS using descriptive statistics, the $\chi^{2}$ test was used to assess for differences in test scores by categorical variables, and differences in age were analyzed first by $\chi^{2}$, and then by independent Student's $t$ test using categorical pass/fail assignment of test scores whereby scores of $75 \%$ or higher were considered passing.

\section{Pilot B Methods}

Pilot B was a randomized experimental study of test-enhanced learning. Patients and/or their primary caregivers, with at least a 2-day hospitalization for an acute stroke diagnosis, were recruited to participate. Patients with National Institutes of Health Stroke Scale (NIHSS) Item 1A and/or Item 9 score greater than 0 were excluded from study enrollment. Consenting subjects were randomized into two groups as follows: target subjects were provided with standardized stroke education over the 2-day period as described above, but were also advised upon enrollment that they would be given a formal written test of stroke knowledge retention just prior to discharge; control subjects were provided with the same standardized education as the target group, but were not notified that there was a formal written testing requirement prior to discharge. Both target and control subjects were administered the same 5-question test (question 1 was only used in pilot B) on the day of discharge (Table 1), which was graded in their presence, and additional education was provided as evidenced by incorrect responses.

Study demographic data were limited to age, sex, race, length of stay, and causative pathogenesis of stroke. Data were entered and analyzed in SPSS using descriptive statistics, and Fisher's exact and MannWhitney $\mathrm{U}$ tests to assess for differences in sample characteristics and test item scores.

\section{Results}

\section{Pilot A Results}

A total of 198 patients and primary caregivers were seen in the Stroke Clinic and consented to completing the written stroke knowledge test. These subjects were seen on average at $4.6 \pm 0.6$ weeks from the time of hospital discharge. Patients were $57 \%$ male, with a mean age of 69 years (range 28-97). Stroke subtypes represented were predominately lacunar (42\%), as well as $20 \%$ large artery atheroma, $16 \%$ cardioembolic, $4 \%$ stroke of other etiology, and $2 \%$ intracerebral hemorrhage; $16 \%$ of patients were deemed cryptogenic. Tests were primarily completed by patients together with their designated primary caregivers (68\%), although $32 \%$ of patients completed the test alone.

Table 2 presents test scores for Pilot A subjects. Overall, only 49 (25\%) subjects scored $100 \%$ correct on the test; 69 (35\%) subjects scored 75\% correct, 49 (25\%) subjects scored 
Table 3. Pilot B subject characteristics

\begin{tabular}{llll}
\hline Characteristic & Control patients & Target patients & $p$ value \\
\hline Female, \% & 53 & 47 & $1.00^{\mathrm{a}}$ \\
African American, \% & 67 & 53 & $0.71^{\mathrm{a}}$ \\
Median age, years & 52 & 64 & $0.03^{\mathrm{b}}$ \\
Ischemic stroke, \% & 80 & 3 & $0.60^{\mathrm{a}}$ \\
Median length of stay, days & 3 & 3 & $0.84^{\mathrm{b}}$ \\
\hline
\end{tabular}

${ }^{\text {a }}$ Fisher's exact test. ${ }^{\mathrm{b}}$ Mann-Whitney U test.

Table 4. Pilot B correct test scores by group

Control patients Target patients $\quad p$ value $^{\mathrm{a}}$

Correct scores by test item, $n$ (\%)

Question 1: Patient's type of stroke Question 2: Signs/symptoms of stroke Question 3: Best response to stroke symptoms Question 4: Personal stroke risk factors Question 5: Personal stroke prevention medications Overall test scores by group, \% (range) Median percent correct for entire test

$\begin{array}{cll}11(73) & 13(87) & 0.65 \\ 8(53) & 11(73) & 0.45 \\ 15(100) & 14(93) & 1.00 \\ 6(40) & 13(87) & 0.02 \\ 10(67) & 15(100) & 0.04 \\ 60(20-100) & 100(40-100) & 0.02\end{array}$

${ }^{\text {a }}$ Fisher's exact test.

$50 \%$ correct, $24(12 \%)$ subjects scored $25 \%$ correct, and 7 (3\%) subjects incorrectly answered all 4 test items. Of note, the test item most commonly graded as incorrect ( $n=117 ; 59 \%$ ) was the patient's or primary caregiver's understanding of his/her own personal risk factors for stroke. Stroke signs and symptoms were incorrectly identified by $74(37 \%)$ subjects, and 52 (26\%) subjects did not understand their medications. Interestingly, 179 (90\%) subjects correctly identified use of the 911 system for new onset symptoms. There were no differences in knowledge retention or any single test item scores by sex, age, stroke mechanism, or patients versus patients/caregivers completing the test.

\section{Pilot B Results}

A convenience sample of 30 subjects consented to participate, with $50 \%$ randomized to each of the target and control groups. Stroke typology, sex, race, and length of stay were similar between groups. However, the target group was significantly older at a median of 64 years (range 35-84) compared to the control group at a median of 52 years (range 24-70; $p=0.03$ ) (Table 3).

Table 4 presents the percent of correct responses among target and control patients for the 5 test items. No between-group differences were noted on items focusing on type of stroke, signs and symptoms of stroke, or use of the 911 system in response to stroke symptoms. However, compared to controls, target subjects had significantly better scores on items dealing with their personal risk factors for stroke (target $87 \%$ vs. control $40 \% ; p=0.02$ ) and understanding their discharge secondary stroke prevention medications (target $100 \%$ vs. control $67 \% ; p=0.04$ ). Overall, total test scores were significantly higher in target group patients compared to control group patients (target median 100\%, range 40-100 correct, vs. 
control median $60 \%$, range $20-100$ correct; $p=0.02$ ). Additionally, target group patients were able to identify significantly more of their personal risk factors (median $100 \%$ correct, range $75-100 \%$ ) compared to control group patients (median $75 \%$ correct, range $0-100 \%$; $p=0.01)$.

\section{Discussion}

Our two pilot studies showed that despite the provision of stroke education by engaged Stroke Team members, patients and primary caregivers fail to retain important information about stroke risk factors, stroke prevention, and symptom recognition, and that use of testenhanced learning methods may improve stroke education retention at hospital discharge. While most patients and primary caregivers were able to identify the importance of using the 911 system for response to stroke, only 37\% (Pilot A) and 53\% (Pilot B controls) were able to correctly identify stroke signs and symptoms, drawing into question whether these subjects would appropriately call 911 should a new stroke event occur.

While stroke education is required during hospitalization $[9,10]$, its ability to affect secondary prevention of stroke and early recognition of new events remains poorly understood. This is especially concerning because provision of high-quality patient education is costly in terms of manpower resources, at a time when few hospitals are willing and able to provide additional full-time nursing staff to support aspects of care beyond actual physical needs. In fact, while hospitals commonly provide written materials to patients and caregivers about stroke [21], it is unclear whether many use highly engaged Stroke Team members to provide the kind of in-depth education used in our pilot studies. While we did not examine how well uneducated patients/primary caregivers would perform on the same test items, it is likely that the results would have been at least similar if not worse compared to our pilot study cohorts, and this draws into question whether providing more than written handouts is necessary, given such poor rates of retention.

Despite poor retention of key stroke knowledge, it is difficult to argue against provision of patient/caregiver education, even with our findings suggesting that only $25 \%$ had retained $100 \%$ of the information provided at 3 months (Pilot A). Perhaps certifying agencies for Stroke Centers err in their ambition to require coverage of such a wide variety of educational topics, at a time when patients and their family members are under significant stress. Interestingly, findings in a sample of over 1,000 subjects from validation of the STROKE Perception Report [23] concluded that overall patient/family knowledge of stroke signs and symptoms was poor at the time of hospital discharge, despite experiencing disabilities associated with an acute stroke event and receiving stroke education, although these same subjects rated provision of stroke education as highly valued in their preparation for discharge [23, 24].

Tests have been shown to enhance learning, by a phenomenon referred to as the "testing effect" [25-29]. While only one study of test-enhanced learning has been reported in patients [29], studies in healthy cohorts demonstrate significant retention of knowledge compared to studying alone [25-28]. We believe that test-enhanced learning may be an important next step in the improvement of knowledge retention and self-management in stroke patients and their caregivers, especially given future mandates for the use of outcome measures (i.e., knowledge retention) by the National Quality Forum as the vetting agency for CMS pay-forperformance metrics. Our Pilot B study demonstrated significant improvement in knowledge retention related to personal risk factors for stroke and the purpose of discharge medications, two of the more complex teaching items required by CMS. Patients' acceptance of responsibility for their health, and competent patient/caregiver self-management are important goals today. Use of test-enhanced learning strategies may improve self-management competencies 
among stroke patients and their caregivers, ultimately increasing adherence to secondary prevention regimens, as well as early recognition and response to future stroke events. Therefore, we propose the Targeted Education in Stroke Trial (TEST) [30] to explore the value of test-enhanced learning in the provision of required stroke education, in an aim to improve patient and primary caregiver knowledge and self-management capabilities following discharge for an acute stroke event.

Our study has limitations that must be acknowledged. First, our Pilot A patients are from a single setting in a primarily White, non-Hispanic community in the Midwest USA. Because of this, we chose not to collect data on race or ethnicity in our study because to do so would increase the risk of personal subject identification and a lack of confidentiality; additionally, differences among groups would not be meaningful given the small numbers representing non-White or Hispanic subjects. Therefore, findings from Pilot A may not be generalizable to settings with diverse racial, ethnic patient populations. Second, we did not compare differences between our cohorts and patients that did not receive our team-delivered stroke education, because this would have forced us to provide a lower standard of care to one group of patients. Therefore, we are unable to conclude whether team-based stroke education was superior to no education. Nonetheless, our findings show that even using our Pilot A methods and Pilot B control methods, stroke knowledge retention in patients and primary caregivers was poor. Lastly, our Pilot B methods used a small sample that prevented us from finding differences in knowledge that may exist by patient characteristics. Nonetheless, Pilot B did find significant and clinically important differences that hold promise for the use of testenhanced teaching methods.

\section{Conclusions}

Provision of stroke education to patients and/or caregivers is a requirement for Stroke Center hospitals, yet may result in very limited retention of stroke knowledge. Methods capable of improving stroke knowledge and self-management competencies are critical to secondary stroke prevention, and both the recognition and response to new stroke symptoms. We aim to evaluate whether test-enhanced learning may be an effective strategy for improving knowledge retention and self-management skills in the TEST study.

\section{Disclosure Statement}

The authors report no conflicts of interest.

\section{References}

1 Berstrom AL, von Koch L, Andersson M, Tham K, Eriksson G: Participation in everyday life and life satisfaction in persons with stroke and their caregivers 3-6 months after onset. J Rehabil Med 2015;47:508-515.

2 Bergstrom AL, Eriksson G, von Koch L, Tham K: Combined life satisfaction of persons with stroke and their caregivers: associations with caregiver burden and the impact of stroke. Health Qual Life Outcomes 2011;9: 1.

3 Carod-Artal FJ, Egido JA: Quality of life after stroke: the importance of a good recovery. Cerebrovasc Dis 2009; 27(suppl 1):204-214.

4 Visser-Meily A, Post M, van de Port I, Maas C, Forstberg-Warleby G, Lindeman E: Psychosocial functioning of spouses of patients with stroke from initial inpatient rehabilitation to 3 years poststroke: course and relations with coping strategies. Stroke 2009:40:1399-1404.

5 Rochette A, Desrosiers J, Bravo G, Tribble DS, Bourget A: Changes in participation level after spouse's first stroke and relationship to burden and depressive symptoms. Cerebrovasc Dis 2007;24:255-260. 
6 Claesson L, Linden T, Skoog I, Blomstrand C: Cognitive impairment after stroke - impact on activities of daily living and costs of care for elderly people. The Goteborg 70+ Stroke Study. Cerebrovasc Dis 2005:19:102-109.

7 Jauch EC, Saver JL, Adams HP Jr, Bruno A, Connors JL, Demaerschalk BM, et al; American Heart Association Stroke Council, Council on Cardiovascular Nursing, Council on Peripheral Vascular Disease, Council on Clinical Cardiology: Guidelines for the early management of patients with acute ischemic stroke: a guideline for healthcare professionals from the American Heart Association/American Stroke Association. Stroke 2013;44: 870-947.

8 American Heart Association: FACTS. Preventable, Treatable, Beatable: Stroke in the United States. Dallas, AHA, 2013.

9 The Joint Commission: Advanced certification for primary stroke centers. www.jointcommission.org/certification/primary_stroke_centers.aspx (accessed June 19, 2015).

10 Centers for Medicare and Medicaid: Hospital clinical quality measures (CQMs). www.cms.gov/Regulationand-Guidance/Legislation/EHRIncentivePrograms/cqm_through_2013.html (accessed June 19, 2015).

11 Centers for Disease Control. http://www.cdc.gov/nchs/data/nhds/2average/2010ave2_firstlist.pdf (accessed June 19, 2015).

12 Healy AF, Boume LE Jr: Empirically valid principles for training in the real world. Am J Psychol 2013;126: 389-399.

13 Lee YS, Vakoch DA: Transfer and retention of implicit ad explicit learning. Br J Psychol 1996;87(Pt 4):637-651.

14 Naik AD, Teal CR, Rodriquez E, Haidet P: Knowing the ABCs: a comparative effectiveness study of two methods of diabetes education. Patient Educ Couns 2011;85:383-389.

15 Mohamed H, Al-Lenjawi B, Amuna P, Zotor F, Elmahdi H: Culturally sensitive patient-centered educational programme for self-anagement of type 2 diabetes: a randomized controlled trial. Prim Care Diabetes 2013;7: 199-206.

16 Cicinello S, Johnston RV Osborne, RH, Wicks I, deKroo T, Clerehan R, O’Neill C, Buchbinder R: Multimedia educational interventions for consumers about prescribed and over-the-counter medications. Cochrane Database Syst Rev 2013;4:CD008416.

17 Forster A, Brown L, Smith J, House A, Knapp P, Wright JJ, Young J: Information provision for stroke patients and their caregivers. Cochrane Database Syst Rev 2012;11:CD001919.

18 Lager KE, Mistri AK, Khunti K, Haunton VJ, Sett AK, Wilson AD: Interventions for improving modifiable risk factor control in the secondary prevention of stroke. Cochrane Database Syst Rev 2014;5:CD009103.

19 Cameron JI, Gignac MA: "Timing It Right": a conceptual framework for addressing the support needs of family caregivers to stroke survivors from the hospital to the home. Patient Educ Couns 2008;70:305-314.

20 Boysen G, Krarup LH, Zeng X, Oskedra A, Korv J, Andersen G, Gluud C, Pedersen A, Lindahl M, Hansen L, Winkel P, Truelsen T; ExStroke Pilot Trial Group: ExStroke Pilot Trial of the effect of repeated instructions to improve physical activity after ischaemic stroke: a multinational randomized controlled clinical trial. Br Med J 2009; 339:b2810.

21 Sullivan KA, Katajamaki A: Stroke education: retention effects in those at low-and high-risk of stroke. Patient Educ Couns 2009;74:205-212.

22 Forster A, Dickerson J, Young J, Patel A, Lalra L, Nixon J, Smithard D, Knapp M, Holloway I, Anwar S, Farrin A; TRACS Trial Collaboration: A structured training programme for caregivers of inpatients after stroke (TRACS): a cluster randomized controlled trial and cost-effectiveness analysis. Lancet 2013;382:2069-2076.

23 Alexandrov AW, Brewer BB, Moore K, Reiner D, Korsnack A, Swatzell V, Johnson B, Nystrom K, Wojner AJ, Alexandrov AV; STROKE Perception Report Investigators: Stroke, Treatment, Risk Factors, Outcome, Knowledge, and Education (STROKE) Perception Report: a prospective, multicenter national validation study of instrument validity and reliability. Stroke 2012;43:ANS1.

24 Boehme AK, Moore K, Reiner D, Korsnack A, Swatzell V, Murphy D, Grau C, Klassman L, Wojner AJ, Alexandrov AW: Patient and family perceptions of the quality of acute stroke services: findings from a multi-center national study of the STROKE Perception Report. Stroke 2012;43:A3537.

25 Baghdady M, Camahan H, Lam EW, Woods NN: Test-enhanced learning and its effect on comprehension and diagnostic accuracy. Med Educ 2014;48:181-188.

26 Larsen DP, Butler AC, Aung WY, Corboy JR, Friedman DI, Sperling MR: The effects of test-enhanced learning on long-term retention in AAN annual meeting courses. Neurology 2015;84:748-754.

27 Larsen DP, Buler AC, Roediger HL 3rd: Comparative effects of test-enhanced learning and self-explanation on long-term retention. Med Educ 2013;47:674-682.

28 Larsen DP, Butler AC, Roediger HR 3rd: Repeated testing improves long-term retention relative to repeated study: a randomized controlled trial. Med Educ 2009;43:1174-1181.

29 Middleton EL, Schwartz MF, Rawson KA, Garvey K: Test-enhanced learning versus errorless learning in aphasia rehabilitation: testing competing psychological principles. J Exp Psychol Learn Mem Cogn 2015;41: 1253-1261.

30 TEST Clinical Investigators. www.teststroke.com (accessed June 19, 2015). 